\title{
A Simple Proof for the Jordan Measurability of Convex Sets
}

\author{
László Szabó \\ László Szabó was born in 1966, in Budapest. He studied mathematics at Eötvös \\ Loránd University, Budapest, and received his Ph.D. from the Hungarian Academy \\ of Sciences in 1996. His research interests are in discrete geometry and convexity.
}

Let $B$ be a coordinate block in $\mathbb{E}^{n}$ of the form

$$
B=\left\{\left(x_{1}, \ldots, x_{n}\right) \in \mathbb{E}^{n} \mid a_{i} \leq x_{i} \leq b_{i},(1 \leq i \leq n)\right\},
$$

where $a_{i}<b_{i}$ for each $i$. Define the volume of $B$ as

$$
V(B)=\left(b_{1}-a_{1}\right)\left(b_{2}-a_{2}\right) \cdots\left(b_{n}-a_{n}\right)
$$

in the obvious way. If $K \subseteq E^{n}$ is a bounded set, let $V^{-}(K)=\sup \sum_{m} V\left(B_{m}\right)$, where the supremum is taken over all packings in $K$ by finite families $\left\{B_{1}, B_{2}, \ldots\right\}$ of blocks, and let $V^{+}(K)=\inf \sum_{m} V\left(B_{m}\right)$, where the infimum is taken over all coverings of $K$ by finite families $\left\{B_{1}, B_{2}, \ldots\right\}$ of blocks. Let us recall that a packing in a set is an arrangement whose members are all contained in the set and have mutually disjoint interiors, and a covering of a set is an arrangement whose union contains the set. It is clear that $V^{-}(K) \leq V^{+}(K)$. Now, we say that the bounded set $K \subseteq E^{n}$ is Jordan measurable if $V^{-}(K)=V^{+}(K)$, and in this case we call this common value the volume of $K$. For a more comprehensive account we refer the reader to the monograph [1]. The aim of this paper is to give a reasonably simple geometric proof (i.e. without using compactness arguments) for the following well-known

Theorem Every bounded convex set $K \subseteq E^{n}$ is Jordan measurable.

Standardbeispiele nicht messbarer Mengen sind wohlbekannt; hingegen sind allgemeine Sätze über die Messbarkeit bestimmter Mengen weniger geläufig. Das Resultat, welches besagt, dass jede beschränkte konvexe Menge des Euklidischen Raumes $\mathbb{E}^{n}$ Jordanmessbar ist, geht wohl auf Minkowski zurück (Volumen und Oberfläche, Math. Ann. 57 (1903), 447-495; Ges. Abh. II, 230-276). László Szabó gibt dafür einen einfachen geometrischen Beweis. ust 
Proof. Bounded convex sets of dimension less than $n$ are clearly Jordan measurable, so we may assume that $K$ is $n$-dimensional. We may also assume, without loss of generality, that $K$ is contained in the cube

$$
C=\left\{\left(x_{1}, \ldots, x_{n}\right) \in \mathbb{E}^{n} \mid-\frac{1}{2} \leq x_{i} \leq \frac{1}{2},(1 \leq i \leq n)\right\} .
$$

By subdividing each edge of $C$ into $2^{j}$ equal parts we can partition $C$ into congruent closed cubes, each of which has edges of length $2^{-j}$. Let $\mathscr{L}_{j}$ denote the family of those small cubes defined above which intersect the interior of $K$, and let $\overline{\mathscr{L}}_{j} \subseteq \mathscr{L}_{j}$ denote the family of those small cubes which intersect the boundary of $K$ as well $(j=1,2, \ldots)$. It is clear that $\cup\left(\mathscr{L}_{j} \backslash \overline{\mathscr{L}}_{j}\right) \subseteq K \subseteq \cup \mathscr{L}_{j}$ for each $j$.

We show that the total volume of the small cubes in $\overline{\mathscr{L}}_{j}$ is not greater than $n 2^{n} 2^{-j}$. Consider the $2^{n}$ directions determined by the $2^{n}$ vectors carrying the vertices of $C$ to the origin $o$. Associate not greater than $n 2^{j(n-1)}$ rays with each direction in the following way. For each vertex $v$ of $C$ consider the rays emanating from the centres of small cubes touching at least one of the $n$ facets of $C$ containing $v$, and having direction vector $\overrightarrow{v o}$. Note that the total number of these rays is not greater than $n 2^{n} 2^{j(n-1)}$ and exactly $2^{n}$ of them pass through the centre of each small cube in $C$.

Let $C^{\prime}$ be an arbitrary small cube in $\overline{\mathscr{L}}_{j}$ and consider the $2^{n}$ rays from the above family which contain the centre of $C^{\prime}$. We claim that at least one of these rays intersects the centre of $C^{\prime}$ before it reaches (according to the natural ordering on the ray) the centre of any other small cube in $\mathscr{L}_{j}$. Indeed, if each of the above rays intersected the centre of some small cube in $\mathscr{L}_{j}$ different from $C^{\prime}$ before reaching the centre of $C^{\prime}$, then choosing one interior point of $K$ in each of these $2^{n}$ small cubes, the convex hull of these interior points of $K$ would contain $C^{\prime}$, so $C^{\prime}$ would not be in $\overline{\mathscr{L}}_{j}$. To see this we need the following simple observation.

Proposition Divide $\mathbb{E}^{n}$ into $2^{n}$ open connected regions by the $n$ coordinate hyperplanes and choose one point in each of these regions. Then the convex hull of these points contains the origin.

Proof of Proposition. The proof is by induction on the dimension $n$. For $n=1$ the assertion is trivial. Assume that we have already proved the result for some $n \geq 1$, and we want to show that it also holds for $n+1$. Let $\mathscr{P}$ denote the set of the points chosen in the regions. Let $\mathscr{P}_{1}$ be the set of those points of $\mathscr{P}$ whose first coordinates are negative. If $\Pi$ denotes the orthogonal projection of the space onto the hyperplane of equation $x_{1}=0$, then by the induction hypothesis the convex hull of $\Pi\left(\mathscr{P}_{1}\right)$ contains the origin. Therefore the convex hull of $\mathscr{P}_{1}$ necessarily contains a point $p_{1}$ of the $x_{1}$-axis with negative first coordinate. Similarly, if $\mathscr{P}_{2}$ denotes the set of those points of $\mathscr{P}$ whose first coordinates are positive, then the convex hull of $\mathscr{P}_{2}$ contains a point $p_{2}$ of the $x_{1}$-axis with positive first coordinate. Now, the origin obviously belongs to the segment $\overline{p_{1} p_{2}}$ and thus to the convex hull of $\mathscr{P}$ as well.

Therefore the number of small cubes in $\overline{\mathscr{L}}_{j}$ is not greater than $n 2^{n} 2^{j(n-1)}$ and thus the total volume of them is at most $n 2^{n} 2^{-j}$. 
The total volumes of small cubes in $\mathscr{L}_{j}$ decrease while the total volumes of small cubes in $\mathscr{L}_{j} \backslash \overline{\mathscr{L}}_{j}$ increase as $j \rightarrow \infty$. Furthermore, as we have seen above, the differences between the two volumes tend to zero as $j \rightarrow \infty$. Hence the two sequences of volumes converge to the same positive number and this number is obviously equal to both $V^{-}(K)$ and $V^{+}(K)$, i.e. $K$ is Jordan measurable.

Remark We note that, apart from the constant $n 2^{n}$, our estimate $n 2^{n} 2^{-j}$ for the total volume of the small cubes in $\overline{\mathscr{L}}_{j}$ is the best possible for each $j$.

Acknowledgment The work was supported by Hung. Nat. Found. for Sci. Research No. F019449

\section{References}

[1] Hadwiger, H.: Vorlesungen über Inhalt, Oberfläche und Isoperimetrie. Springer Verlag, Berlin, 1957

László Szabó

Computer and Automation Institute

Hungarian Academy of Sciences

1111 Budapest

Lágymányosi út 11, Hungary 